\title{
Dual PI3K/mTOR inhibitor NVP-BEZ235-induced apoptosis of hepatocellular carcinoma cell lines is enhanced by inhibitors of autophagy
}

\author{
ZHEXING CHANG $^{1 *}$, GUANG SHI $^{2 *}$, JIQIANG JIN $^{3}$, HUATAO GUO $^{4}$, XUEFENG GUO $^{5}$, \\ FANGYUE LUO ${ }^{6}$, YUGUO SONG ${ }^{7}$ and XIAOJING JIA ${ }^{8}$
}

\author{
${ }^{1}$ Department of Oncology, Beihua Affiliated Hospital, Jilin 132000; ${ }^{2}$ Department of Oncology, The Second Hospital of \\ Jilin University, Changchun, Jilin 130041; ${ }^{3}$ The Second Group of Oncology, Jilin Central Hospital; Departments of \\ ${ }^{4}$ Cardiology, ${ }^{5}$ Emergency, ${ }^{6}$ Pediatrics, ${ }^{7}$ Immunity, Beihua Affiliated Hospital, Jilin 132000; ${ }^{8}$ Department of \\ Tumor Radiotherapy, The Second Hospital of Jilin University, Changchun, Jilin 130041, P.R. China
}

Received December 21, 2012; Accepted March 8, 2013

DOI: $10.3892 / \mathrm{ijmm} .2013 .1351$

\begin{abstract}
Dysregulation of the phosphoinositide 3-kinase $(\mathrm{PI} 3 \mathrm{~K}) / \mathrm{AKT} / \mathrm{mammalian}$ target of rapamycin (mTOR) signaling has been found in several types of human cancer, including hepatocellular carcinoma (HCC). NVP-BEZ235 is a novel, orally bioavailable dual PI3K/mTOR inhibitor that has exhibited promising activity against $\mathrm{HCC}$ in preclinical models. Autophagy is a cellular lysosomal degradation pathway essential for the regulation of cell survival and death to maintain homeostasis. This process is negatively regulated by mTOR signaling and often counteracts the efficacy of certain cancer therapeutic agents. In this study, we explored the role of autophagy in apoptosis induced by NVP-BEZ235 in two HCC cell lines, Hep3B and PLC/PRF/5, and identified the mechanism of combinatorial treatment. NVP-BEZ235 was effective in inhibiting the growth of the two HCC cell lines possibly though induction of apoptosis. NVP-BEZ235 also potently increased the expression of LC3-II and decreased the expression of p62, indicating induction of autophagy. When NVP-BEZ235 was used in combination with Atg5 siRNA or the autophagy inhibitor 3-methyladenine (3-MA), enhancement of the inhibitory effects on the growth of HCC cells was detected. In addition, enhanced induction of apoptosis was observed in cells exposed to the combination of NVP-BEZ235 and Atg5 siRNA or 3-MA. Thus, induction of autophagy by NVP-BEZ235 may be a survival mechanism
\end{abstract}

Correspondence to: Professor Xiaojing Jia, Department of Tumor Radiotherapy, The Second Hospital of Jilin University, 218 Ziqiang Street, Changchun 130041, P.R. China

E-mail: jiaxiaojing@yahoo.com.cn

*Contributed equally

Key words: hepatocellular carcinoma, PI3K/AKT/mTOR, NVP-BEZ235, autophagy, apoptosis that counteracts its anticancer effects. Based on these data, we suggest a strategy to enhance the anticancer efficacy of BEZ235 by blockade of autophagy. Thus, our study provides a rationale for the clinical development of combinations of NVP-BEZ235 and autophagy inhibitors for the treatment of $\mathrm{HCC}$ and other malignancies.

\section{Introduction}

Human hepatocellular carcinoma (HCC) is the fifth most common malignancy worldwide, and is the third leading cause of cancer-related mortality. The incidence and mortality of HCC is inhomogenous in undeveloped and developed countries, and the majority of HCC occurs in Asia and Africa. However, its incidence has increased rapidly in the past two decades in Western Europe $(1,2)$. Despite the magnitude of the disease, existing treatments are of limited efficacy. Less than $30 \%$ of the patients are suitable for curative treatment, and the recurrence is a frequent issue following tumor ablation (3). Accordingly, new novel therapeutic approaches for HCC are urgently required.

Among the molecular mechanisms of HCC, several signaling pathways play a vital role in hepatocarcinogenesis (4), and the phosphoinositide 3-kinase (PI3K)/AKT/mammalian target of rapamycin (mTOR) signaling is aberrantly activated in HCC. The PI3K/AKT/mTOR signaling pathway has been the focus of research in recent years due to its potential role in cancer $(5,6)$. The PI3K/AKT/mTOR signaling pathway acts as a convergence for numerous upstream signals and stimulates the activity of a multitude of downstream effectors, thereby mediating cellular survival, growth (5). Therefore, it is not surprising that aberrant and deregulation activation of this signaling is a common molecular event in several types of malignancy (6). These insights have resulted in the development of novel therapies targeting constituents of this pathway (single or multiple PI3K/mTOR inhibitors) and currently several inhibitors are in clinical trials (7-9). Therefore, targeting the PI3K/AKT/mTOR signaling has become the novel therapeutic approach for human HCC treatment (9). 
Autophagy is a cellular lysosomal degradation pathway that is essential for regulation of cell survival and death to maintain cellular homeostasis $(10,11)$. One of the key regulators of autophagy is mTOR, which is the major inhibitory signal that shuts off autophagy in the presence of growth factors and abundant nutrients (11). Accordingly, inhibition of mTOR signaling (e.g., by the mTOR inhibitor rapamycin) induces autophagy (12). Autophagy can be either a pro-survival or death mechanism depending on the circumstances $(10,11)$, thereby generating a variable impact on the outcome of cancer therapy. In the present study, we investigated whether inhibition of autophagy would enhance the apoptosis of HCC cells.

We focused on the role of NVP-BEZ235, the effective $\mathrm{PI} 3 \mathrm{~K} / \mathrm{mTOR}$ dual inhibitor on cancer therapy (13-15) on the induction of apoptosis in human HCC cell lines and explored the impact of autophagy induction on its anticancer activity against HCC. We also examined if combination of autophagy inhibitors with NVP-BEZ235 could enhance the apoptosis of HCC cells.

\section{Materials and methods}

Materials. 3-(4,5-dimethylthiazol-2-yl)-2,5-diphenyltetrazolium bromide (MTT) and 3-methyladenine (3-MA) were purchased from Sigma. Atg5 siRNA plasmid was purchased from Santa Cruz Biotechnology, Inc., Santa Cruz, CA, USA (sc-29918). Dulbecco's modified Eagle's medium (DMEM) and fetal bovine serum (FBS) were purchased from Gibco. The antibodies anti-caspase-3, anti-cleaved caspase-3, anti-PARP, anti-LC3, anti-Atg5, anti-AKT, anti-p-AKT, anti-p70S6K, anti-p-p70S6K and anti-p62 were purchased from Santa Cruz Biotechnology, Inc. NVP-BEZ235 was purchased from LC Laboratories.

Cell culture. The human Hep3B and PLC/PRF/5 cells were cultured in DMEM with 10\% FBS, under standard culture conditions $\left(37^{\circ} \mathrm{C}\right.$ and $\left.5 \% \mathrm{CO}_{2}\right)$.

Cell viability assays. Hep3B and PLC/PRF/5 cells were cultured in 96-well plates at a density of $1 \times 10^{4}$ cells/well in $100 \mu 1$ of complete medium. Each group was repeated in nine separate wells. MTT reagent $[12 \mu 1,5 \mathrm{mg} / \mathrm{ml}$ in phosphatebuffered saline (PBS)] was added to each well for 4-6 h. Following treatment, each well was dissolved in $150 \mu \mathrm{l}$ DMSO. Absorbance was recorded at a wavelength of $490 \mathrm{~nm}$.

Western blot analysis. Hep3B and PLC/PRF/5 cells were washed with cold PBS and then $300 \mu 1$ radioimmunoprecipitation (RIPA) buffer [50 mM Tris- $\mathrm{HCl}$ (pH 6.8), 0.1\% SDS, $150 \mathrm{mM} \mathrm{NaCl}, 1 \mathrm{mM}$ EDTA, $0.1 \mathrm{mM} \mathrm{Na}_{3} \mathrm{VO}_{4}, 1 \mathrm{mM}$ sodium fluoride (NaF), $1 \%$ Triton X-100, $1 \%$ NP-40, 1 mM dithiothreitol, and $1 \mathrm{mM}$ PMSF, $1 \mu \mathrm{g} / \mathrm{ml}$ aprotinin, $1 \mu \mathrm{g} / \mathrm{ml}$ leupeptin, $1 \mu \mathrm{g} / \mathrm{ml}$ pepstatin A] were added to each group. Cell lysates were then shaken in a cold room $\left(4^{\circ} \mathrm{C}\right)$ for $30 \mathrm{~min}$ and centrifuged at $14,000 \mathrm{x} \mathrm{g}$ for $10 \mathrm{~min}$. Protein concentrations in the supernatants were detected using the BCA protein assay. For western blot analysis, $50 \mu \mathrm{g}$ proteins were separated by $12 \%$ (w/v) SDS-polyacrylamide gel electrophoresis. After running gels, proteins were transferred onto PVDF membranes and were then blocked with $5 \%(\mathrm{w} / \mathrm{v})$ skim milk in buffer $[10 \mathrm{mM}$
Tris-HCl (pH 7.6), $100 \mathrm{mM} \mathrm{NaCl}$, and $0.1 \%$ (v/v) Tween-20] for $2 \mathrm{~h}$ at room temperature $\left(25^{\circ} \mathrm{C}\right)$; the primary antibodies were added overnight on the shaker in a cold room. Then, PVDF membranes were incubated with secondary antibodies (Sigma) for $1 \mathrm{~h}$ at room temperature. The semi-quantitation of proteins was surveyed with a Tanon GIS gel imager system.

Atg 5 siRNA transfection. Prior to transfection, 50\% Hep3B and $\mathrm{PLC} / \mathrm{PRF} / 5$ cells were grown in each dish. These cells were transfected with $50 \mathrm{nmol} / \mathrm{l}$ of siRNA using Lipofectamine RNAiMAX (Invitrogen, Carlsbad, CA, USA) according to the manufacturer's protocol. Cells were harvested for western blotting at $24-48 \mathrm{~h}$ post transfection.

Mitochondrial membrane potential (MMP) analysis. To detect the change of MMP, we used the JC-1 staining through flow cytometry. The assay was performed according to the manufacturer's instructions (Invitrogen). Briefly, Hep3B and $\mathrm{PLC} / \mathrm{PRF} / 5$ cells were trypsinized, washed with PBS twice, and resuspended in PBS at a concentration of $1-1.5 \times 10^{6} \mathrm{cells} / \mathrm{ml}$. Hep3B and PLC/PRF/ 5 cells were then stained with $3 \mu 1$ of JC- 1 $(1 \mathrm{mg} / \mathrm{ml})$ and incubated in the dark at $37^{\circ} \mathrm{C}$ for $1 \mathrm{~h}$. The JC- 1 positive cells were subsequently detected by a FACSCalibur flow cytometer.

Statistical analysis. The data were analyzed by $\mathrm{t}$-test. $\mathrm{P}<0.05$ was considered to represent a statistically significant difference. Data are representative of three independent experiments performed in triplicate.

\section{Results}

NVP-BEZ235 (NVP) inhibits growth and the PI3K/mTOR pathway of the HCC cell lines Hep3B and PLC/PRF/5. Overexpression of PI3K/AKT/mTOR signaling has been reported in several types of cancer, including $\operatorname{HCC}(5,9)$. Targeting the PI3K/AKT/mTOR signal pathway may be the novel therapeutic approach for HCC. NVP-BEZ235 is a novel, orally bioavailable dual PI3K/mTOR inhibitor that has exhibited promising activity in preclinical models $(13,14)$. First, we used MTT to detect the effect of NVP on the two HCC cell lines, Hep3B and PLC/PRF/5. NVP decreased the cell viability of the two cell lines in a dose-dependent manner (Fig. 1A and B). We also detected the expression of PI3K/ mTOR pathway proteins in the two cell lines treated with NVP. NVP downregulated the expression of p-AKT and p-p70S6K (Fig. 1C and D).

NVP induces apoptosis in Hep3B and PLC/PRF/5 cells. Based on the above results, we further detected the apoptosisrelated proteins in Hep3B and PLC/PRF/5 treated with NVP. We detected the apoptotic relative proteins caspase- 3 and PARP. NVP increased the expression of cleaved caspase-3 and cleaved PARP in Hep3B (Fig. 2A and B). Similar results can be seen in another HCC cell line, PLC/PRF/5 (Fig. 2C and D). We next measured the MMP in Hep3B and PLC/PRF/5 cells. NVP clearly induced the loss of the MMP in Hep3B and PLC/ PRF/5 cells (Fig. 2E and F). These results indicate that NVP induces apoptosis in Hep3B and PLC/PRF/5 cells, possibly through the mitochondrial apoptotic pathway. 


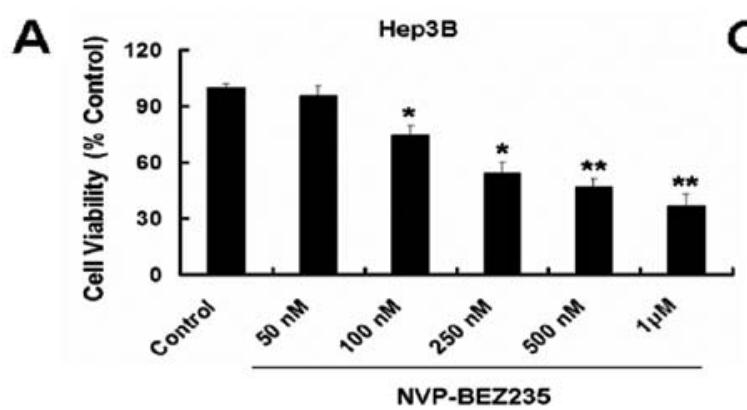

C
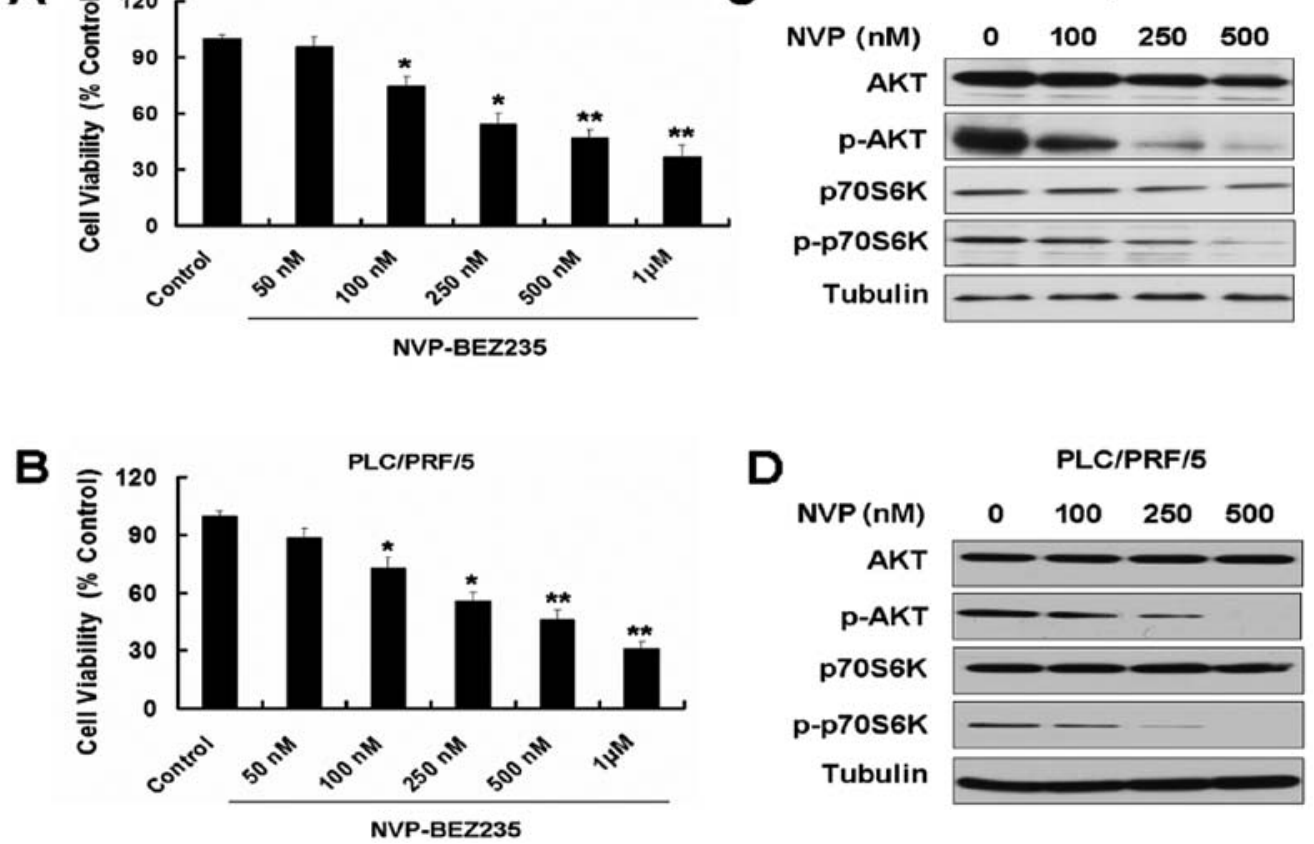

Figure 1. NVP-BEZ235 (NVP) inhibits growth and the PI3K/mTOR pathway of the hepatocellular carcinoma (HCC) cell lines Hep3B and PLC/PRF/5. (A) Hep3B cells were treated with 50, 100, 250,500 and 1,000 nM NVP for $24 \mathrm{~h}$. Cell viability was determined by MTT assay. Data are presented as the means $\pm \mathrm{SD}, \mathrm{n}=9$. (B) PLC/PRF/5 cells were treated with 50, 100, 250, 500 and 1,000 nM NVP for $24 \mathrm{~h}$. Cell viability was determined by MTT assay. Data are presented as the means $\pm \mathrm{SD}, \mathrm{n}=9$. ${ }^{*} \mathrm{P}<0.05$ vs. control group; ${ }^{* *} \mathrm{P}<0.01$ vs. control group. (C) Western blot analysis for the expression of AKT, p-AKT, p70S6K and p-p70S6K in Hep3B cells treated with 100, 250 and $500 \mathrm{nM} \mathrm{NVP}$ for $24 \mathrm{~h}$. (D) Western blot analysis for the expression of AKT, p-AKT, p70S6K and p-p70S6K in PLC/PRF/5 cells treated with 100, 250 and $500 \mathrm{nM} \mathrm{NVP}$ for $24 \mathrm{~h}$.
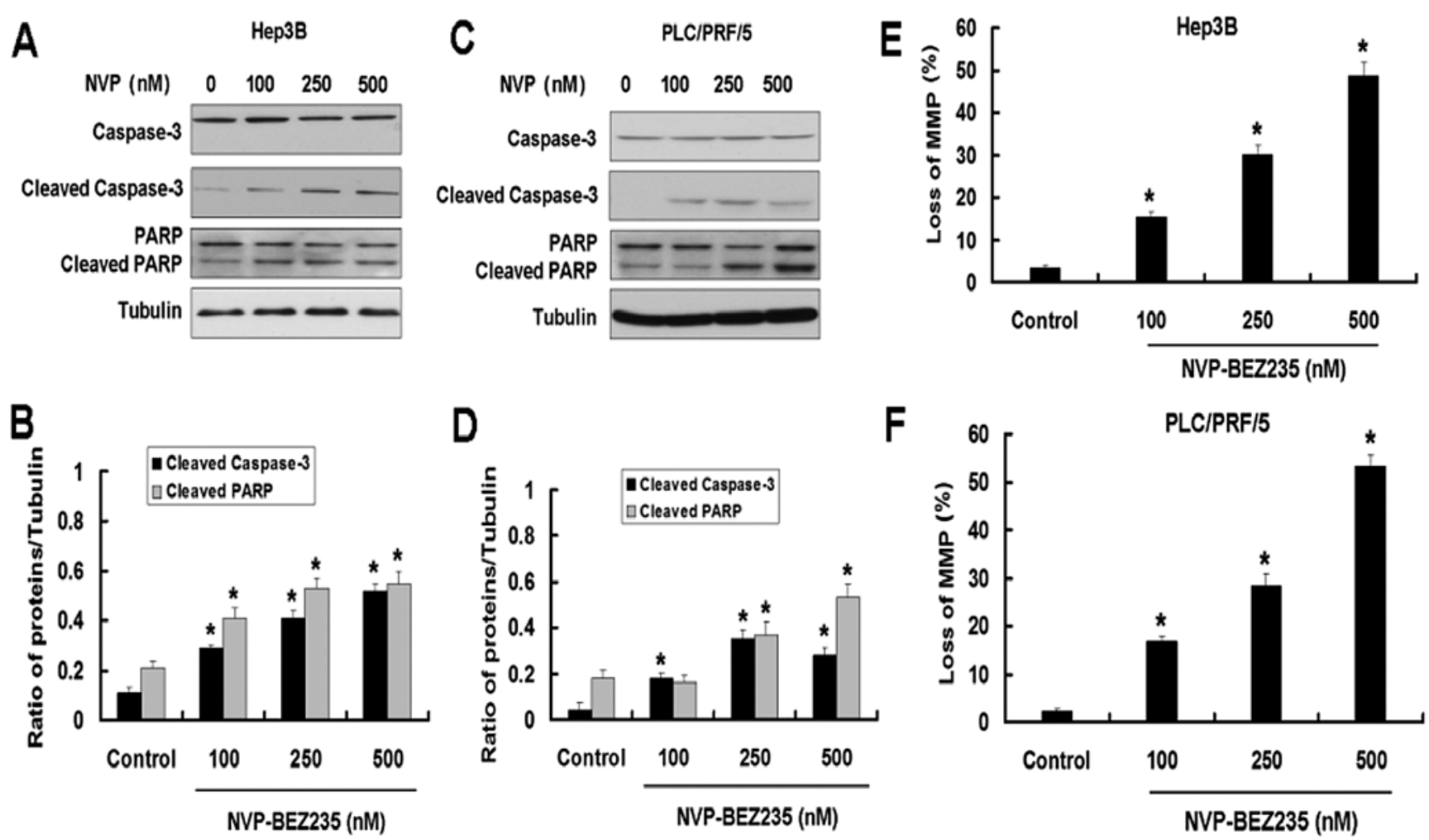

Figure 2. NVP induces apoptosis in Hep3B and PLC/PRF/5 cells. (A) Western blot analysis for the expression of caspase-3, cleaved caspase-3 and PARP in Hep3B cells treated with 100, 250 and $500 \mathrm{nM}$ NVP for $24 \mathrm{~h}$. (B) Quantitation of cleaved caspase-3 and cleaved PARP protein levels in Hep3B cells treated with 100, 250 and $500 \mathrm{nM} \mathrm{NVP}$ for $24 \mathrm{~h}$. (C) Western blot analysis for the expression of caspase-3, cleaved caspase-3 and PARP in PLC/PRF/5 cells treated with 100, 250 and $500 \mathrm{nM} \mathrm{NVP}$ for $24 \mathrm{~h}$. (D) Quantitation of cleaved caspase- 3 and cleaved PARP protein levels in PLC/PRF/5 cells treated with 100,250 and $500 \mathrm{nM} \mathrm{NVP}$ for $24 \mathrm{~h}$. (E) Hep3B cells treated with 100, 250 and $500 \mathrm{nM} \mathrm{NVP}$ for $24 \mathrm{~h}$. The cells were harvested following treatment and were stained with JC-1. (F) PLC/PRF/5 cells treated with 100, 250 and $500 \mathrm{nM}$ NVP for $24 \mathrm{~h}$. The cells were harvested following treatment and were stained with JC-1. Data are presented as the means $\pm \mathrm{SD}, \mathrm{n}=3 .{ }^{*} \mathrm{P}<0.05$ vs. control group. 

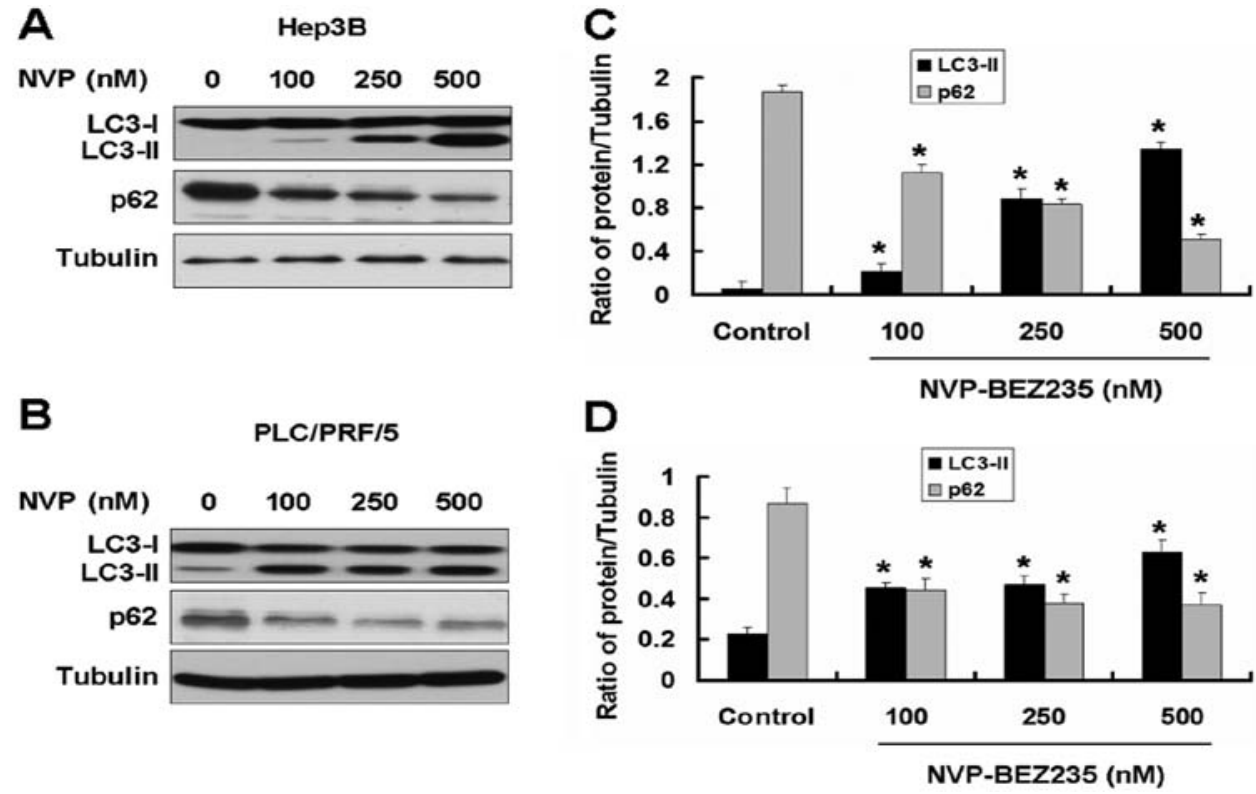

Figure 3. NVP induces autophagy in Hep3B and PLC/PRF/5 cells. (A) Western blot analysis for the expression of LC3 and p62 in Hep3B cells treated with 100,250 and $500 \mathrm{nM} \mathrm{NVP}$ for $24 \mathrm{~h}$. (B) Western blot analysis for the expression of LC3 and p62 in PLC/PRF/5 cells treated with 100, 250 and $500 \mathrm{nM}$ NVP for 24 h. (C) Quantitation of LC3-II and p62 protein levels in Hep3B cells. (D) Quantitation of LC3-II and p62 protein levels in PLC/PRF/5. Data are presented as the means $\pm \mathrm{SD}, \mathrm{n}=3$. ${ }^{*} \mathrm{P}<0.05$ vs. control group.

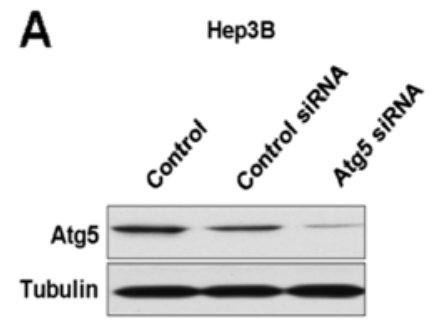

B

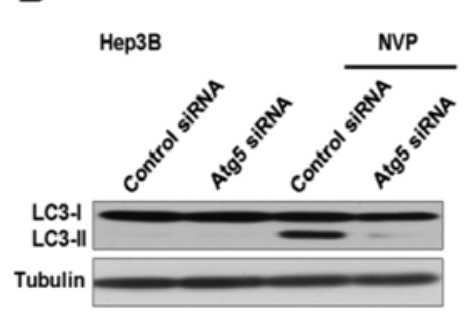

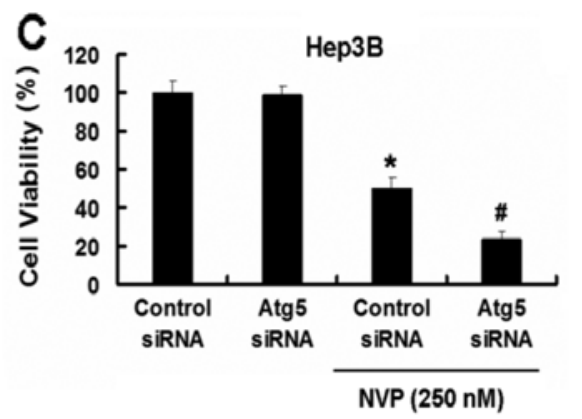

D

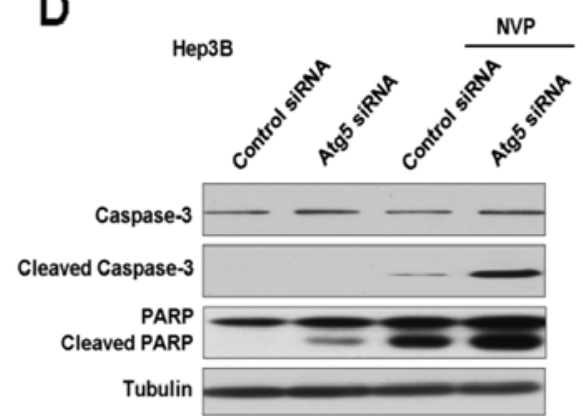

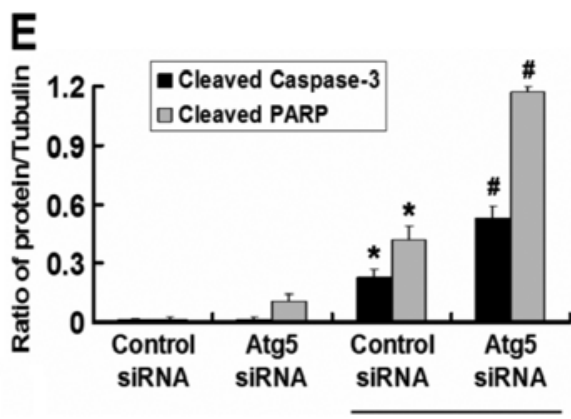

$\mathbf{F}$

NVP (250 nM)

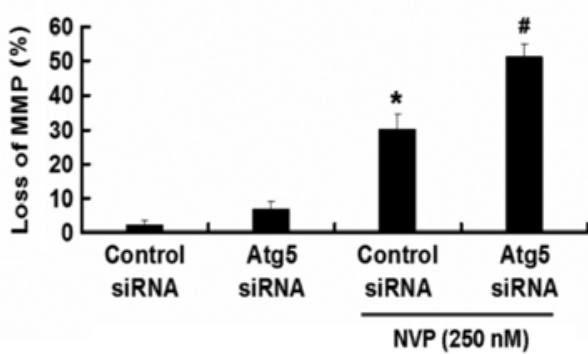

Figure 4. Inhibition of autophagy by Atg5 siRNA enhances apoptosis induced by NVP in Hep3B cells. (A) Western blot analysis for the expression of Atg5 in Hep3B cells treated with control siRNA or Atg5 siRNA. (B) Western blot analysis for the expression of LC3 in Hep3B cells treated with control siRNA or Atg5 siRNA with or without NVP $(250 \mathrm{nM}, 24 \mathrm{~h})$. (C) Hep3B cells treated with control siRNA or Atg5 siRNA with or without NVP for $24 \mathrm{~h}$. Cell viability was determined by MTT assay. Data are presented as the means $\pm S D, n=9$. (D) Western blot analysis for the expression of caspase-3, cleaved caspase-3 and PARP in Hep3B cells treated with control siRNA or Atg5 siRNA with or without NVP ( $250 \mathrm{nM}, 24 \mathrm{~h})$. (E) Quantitation of cleaved caspase-3 and cleaved PARP protein levels. (F) Hep3B cells treated with control siRNA or Atg5 siRNA with or without NVP $(250 \mathrm{nM}, 24 \mathrm{~h})$. The cells were harvested following treatment and were stained with JC-1. Data are presented as the means $\pm \mathrm{SD}, \mathrm{n}=3$. ${ }^{*} \mathrm{P}<0.05$ vs. control group; ${ }^{\mathrm{P}} \mathrm{P}<0.05$ vs. NVP group.

NVP induces autophagy in Hep3B and PLC/PRF/5 cells. Aside from the effect on tumor cell growth, the PI3K/AKT/mTOR pathway is also involved in autophagy. We further detected the autophagy-related proteins in Hep3B and PLC/PRF/5 cells treated with NVP. Microtubule-associated protein LC3 (the mammalian equivalent of yeast ATG8) and p62 are the two 
A

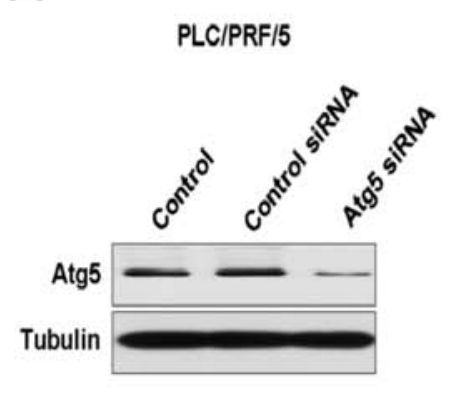

B

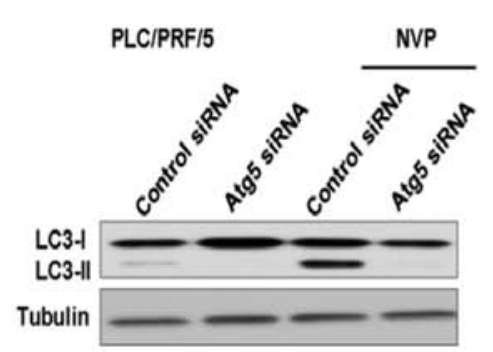

C

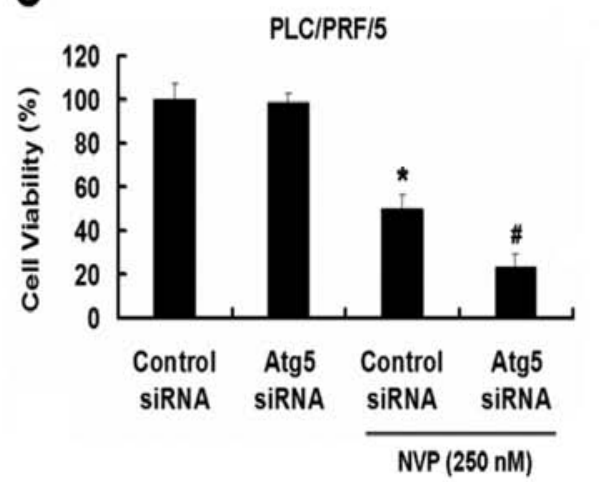

$\operatorname{NVP}(250 \mathrm{nM})$

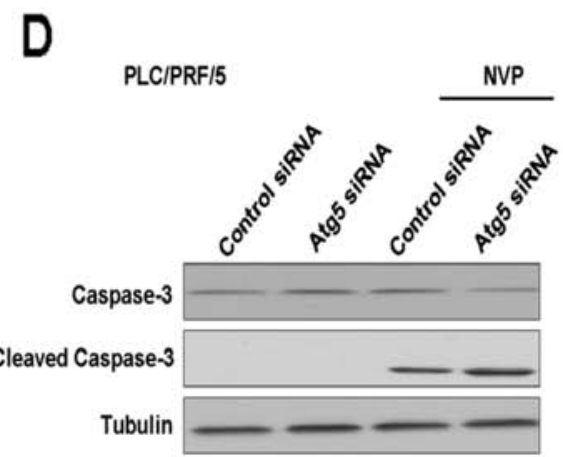

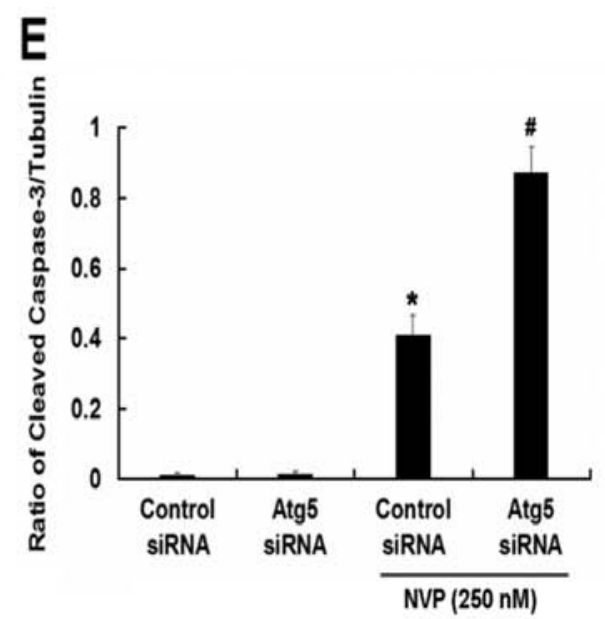

$\mathbf{F}$

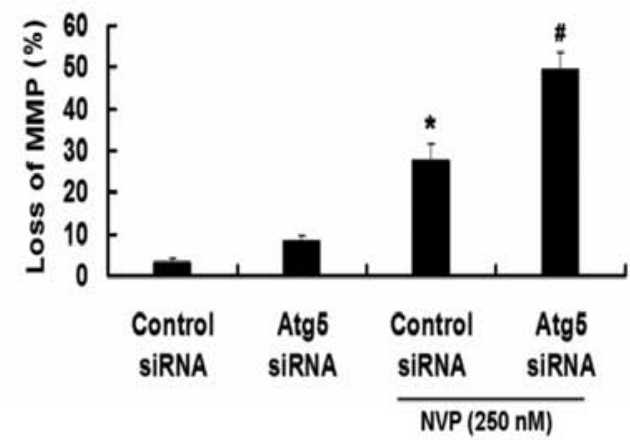

Figure 5. Inhibition of autophagy by Atg5 siRNA enhances apoptosis induced by NVP in PLC/PRF/5 cells. (A) Western blot analysis for the expression of Atg5 in PLC/PRF/5 cells treated with control siRNA or Atg5 siRNA. (B) Western blot analysis for the expression of LC3 in PLC/PRF/5 cells treated with control siRNA or Atg5 siRNA with or without NVP (250 nM, $24 \mathrm{~h})$. (C) PLC/PRF/5 cells treated with control siRNA or Atg5 siRNA with or without NVP for $24 \mathrm{~h}$. Cell viability was determined by MTT assay. Data are presented as the means \pm SD, $n=9$. (D) Western blot analysis for the expression of caspase-3 and cleaved caspase-3 in PLC/PRF/5 cells treated with control siRNA or Atg5 siRNA with or without NVP ( $250 \mathrm{nM}, 24 \mathrm{~h})$. (E) Quantitation of cleaved caspase-3 protein level. (F) PLC/PRF/5 cells treated with control siRNA or Atg5 siRNA with or without NVP ( $250 \mathrm{nM}, 24 \mathrm{~h})$. The cells were harvested following treatment and were stained with JC-1. Data are presented as the means $\pm \mathrm{SD}, \mathrm{n}=3$. ${ }^{*} \mathrm{P}<0.05$ vs. control group; ${ }^{\sharp} \mathrm{P}<0.05$ vs. NVP group.

markers for autophagy. There are two cellular forms of LC3, LC3-I and LC3-II. LC3-I converts to LC3-II when autophagy occurs and the amount of LC3-II becomes a marker for the formation of autophagosomes. Following the occurrence of autophagy, the expression of p62 decreased. We used western blotting to detect the expression of the two proteins in Hep3B and PLC/PRF/5 cells treated with NVP. NVP enhanced the expression of LC3-II and decreased the expression of p62 in Hep3B and PLC/PRF/5 cells, indicating that NVP induces autophagy in Hep3B and PLC/PRF/5 cells (Fig. 3).

Inhibition of autophagy by Atg5 siRNA enhances apoptosis induced by NVP in Hep $3 B$ and PLC/PRF/5 cells. As shown in the results above, NVP induces apoptosis and autophagy in HCC cell lines. Autophagy is always considered a protection process when cells are under low nutrition. However, the role of autophagy in apoptosis induced by chemicals is uncertain. We first used the Atg5 siRNA plasmid to explore the role of autophagy in apoptosis induced by NVP. Atg5 siRNA inhibited the expression of Atg5 and the increase of LC3-II induced by NVP in Hep3B cells (Fig. 4A and B). Inhibition of autophagy by $\operatorname{Atg} 5$ siRNA intensified the growth inhibition of Hep3B cells induced by NVP (Fig. 4C). Meanwhile, Atg5
siRNA further increased the apoptotic relative proteins cleaved caspase- 3 and cleaved PARP induced by NVP in Hep3B cells (Fig. 4D and E). Atg5 siRNA also increased the loss of MMP in Hep3B cells induced by NVP (Fig. 5F). Similar results were found in the other HCC cell line, PLC/PRF/5 (Fig. 5).

Inhibition of autophagy by autophagy inhibitor 3-MA enhances apoptosis induced by NVP in Hep $3 B$ and PLC/PRF/5 cells. We further explored the role of autophagy in apoptosis induced by NVP. We used the autophagy inhibitor 3-MA to analyze the effect of autophagy on apoptosis in Hep3B and PLC/PRF/5 cells induced by NVP. The autophagy inhibitor 3-MA inhibits the autophagy induced by NVP (Fig. 6A). As the Atg5 siRNA, 3-MA further augmented the decrease of cell viability induced by NVP in Hep3B cells. Combination of NVP and 3-MA further increased the expression of apoptotic relative proteins cleaved caspase- 3 and cleaved PARP and the loss of MMP induced by NVP in Hep3B cells. Similar results were observed in PLC/PRF/5 cells (Fig. 7).

These results indicate that autophagy plays a key role in apoptosis induced by NVP. Combination of NVP and autophagy inhibition may be a novel strategy for the treatment of HCC. 

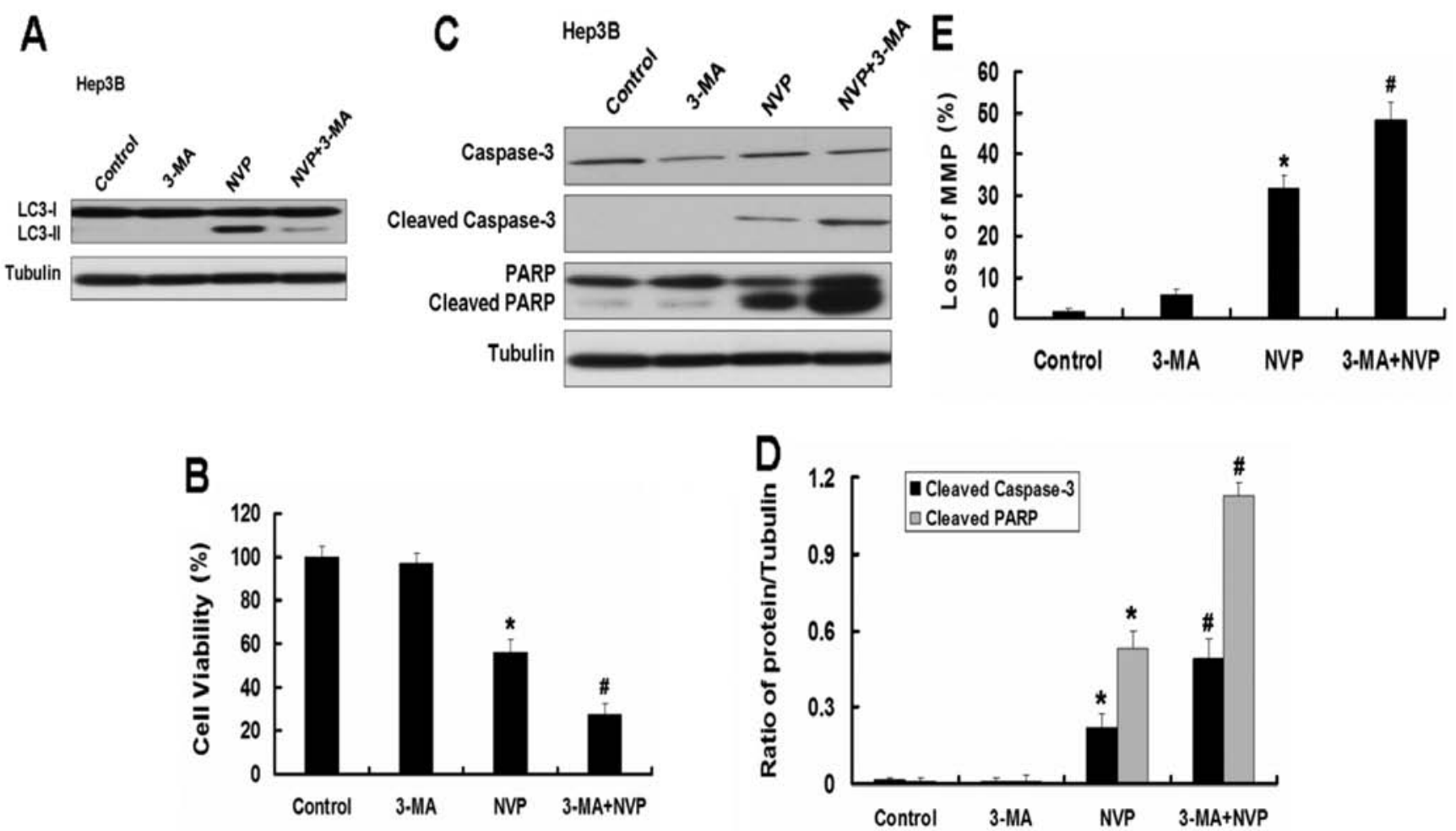

Figure 6. Inhibition of autophagy by autophagy inhibitor 3-MA enhances apoptosis induced by NVP in Hep3B cells. (A) Western blot analysis for the expression of LC3 in Hep3B cells treated with NVP (250 nM), 3-MA or NVP and 3-MA for 24 h. (B) Hep3B cells treated with NVP (250 nM), 3-MA or NVP and 3-MA for $24 \mathrm{~h}$. Cell viability was determined by MTT assay. Data are presented as the means $\pm \mathrm{SD}, \mathrm{n}=9$. (C) Western blot analysis for the expression of caspase-3, cleaved caspase-3 and PARP in Hep3B cells treated with NVP (250 nM), 3-MA or NVP and 3-MA for 24 h. (D) Quantitation of cleaved caspase-3 and cleaved PARP protein levels. (E) Hep3B cells treated with NVP $(250 \mathrm{nM}), 3-\mathrm{MA}$ or NVP and 3-MA for $24 \mathrm{~h}$. The cells were harvested following treatment and were stained with JC-1. Data are presented as the means $\pm \mathrm{SD}, \mathrm{n}=3 .{ }^{*} \mathrm{P}<0.05$ vs. control group; ${ }^{*} \mathrm{P}<0.05$ vs. NVP group.

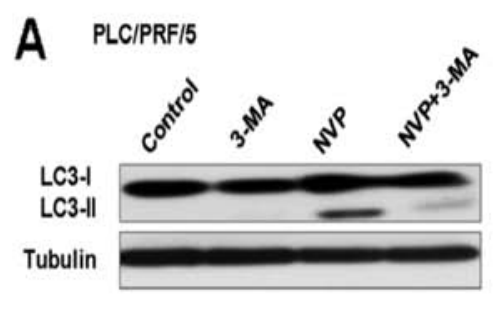

B

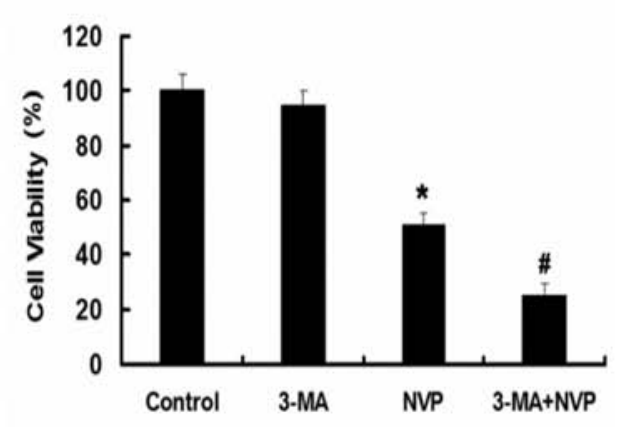

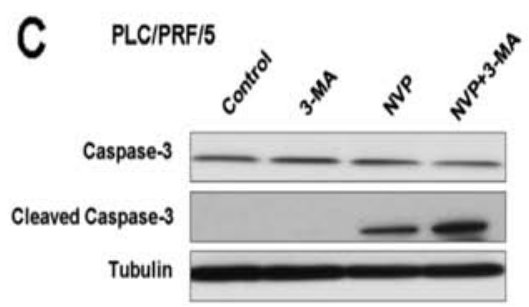

D

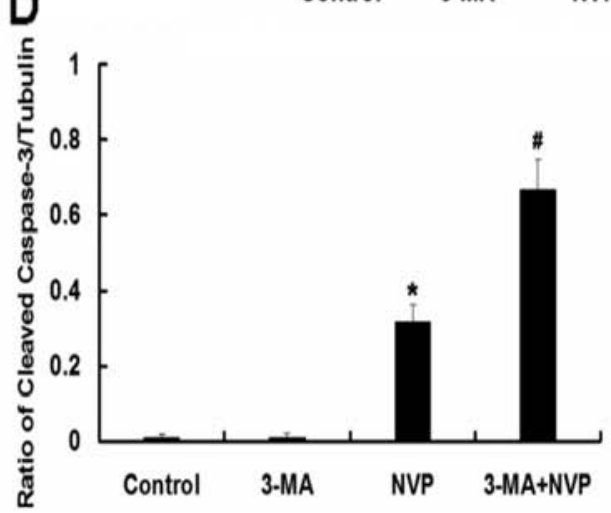

Figure 7. Inhibition of autophagy by autophagy inhibitor 3-MA enhances apoptosis induced by NVP in PLC/PRF/5 cells. (A) Western blot analysis for the expression of LC3 in PLC/PRF/5 cells treated with NVP ( $250 \mathrm{nM}), 3-\mathrm{MA}$ or NVP and 3-MA for $24 \mathrm{~h}$. (B) PLC/PRF/5 cells treated with NVP (250 nM), 3-MA or NVP and 3-MA for $24 \mathrm{~h}$. Cell viability was determined by MTT assay. Data are presented as the means \pm SD, $n=9$. (C) Western blot analysis for the expression of caspase-3 and cleaved caspase-3 by NVP ( $250 \mathrm{nM}), 3-\mathrm{MA}$ or NVP and 3-MA for $24 \mathrm{~h}$. (D) Quantitation of cleaved caspase-3 protein level. (E) PLC/PRF/5 cells treated with NVP (250 nM), 3-MA or NVP and 3-MA for $24 \mathrm{~h}$. The cells were harvested following treatment and were stained with JC-1. Data are presented as the means $\pm \mathrm{SD}, \mathrm{n}=3$. ${ }^{*} \mathrm{P}<0.05$ vs. control group; ${ }^{\#} \mathrm{P}<0.05$ vs. NVP group. 


\section{Discussion}

$\mathrm{HCC}$ is a fatal cancer for men and women and is caused by complicated risk factors (16). Due to frequent de novo and acquired resistance of HCC to chemotherapy, the effective options for therapy of HCC are limited $(17,18)$. Consequently, there is a strong interest in identifying novel molecular targets for therapy of HCC.

Taking into account the role of PI3K/AKT/mTOR signaling in HCC cellular survival, negating apoptosis (19) would indicate that a marked proapoptotic response to the inhibition of this signaling pathway may be expected. However, as previously found (20), apoptosis is not necessarily the primary response to PI3K/AKT/mTOR inhibition, particularly in cancer cells where marked apoptosis suppression may be the consequence of multiple genetic alterations. The current study suggests that the PI3K/AKT/mTOR inhibitors induce productive autophagy in cancer cells $(21,22)$. Jung et al $(23)$ reported that mTOR is a master regulator of autophagy through the phosphorylation of its downstream gene ULK. Thus, it is not surprising that mTOR blockade would lead to autophagy induction.

Autophagy induced by the blockade of the PI3K/AKT/ mTOR pathway has been identified in several studies as a mechanism of cell death, whereas other articles have provided data showing the role of this process in therapeutic resistance. Recent examples of the former include the finding that PI3K/ AKT/mTOR pathway inhibition increases radiosensitivity by augmenting autophagic response (24), and that combining the dual PI3K/mTOR inhibitor NVP-BEZ235 with the mTORC1 inhibitor temsirolimus leads to cell death secondary to massive autophagic response (25). On the contrary, autophagy blockade has been indicated as enhancing the proapoptotic effects of $\mathrm{PI} 3 \mathrm{~K} / \mathrm{mTOR}$ inhibitors in preclinical cancer models $(21,22)$.

Apoptosis and autophagy are significantly involved in cancer. Both apoptosis and autophagy play important roles in the development, cellular homeostasis and, particularly, in the oncogenesis of mammals. The mechanisms of apoptosis and autophagy are different and involve fundamentally distinct sets of regulatory and executioner molecules (26-28). The crosstalk between apoptosis and autophagy is therefore complex, sometimes even contradictory, in nature, yet it is critical to the overall fate of the cell (29). In some cellular settings, autophagy serves as a cell survival pathway to suppress apoptosis (30). On the other hand, autophagy could lead to cell death, either in collaboration with apoptosis or as a back-up mechanism when apoptosis is defective (29). Recent studies have shown that autophagy may play an important role in the regulation of cancer development and progression. Whether autophagy represents a mechanism for resisting apoptosis or a mechanism for initiating a nonapoptotic form of programmed cell death remains unclear (31-33).

Thus, we hypothesize that inhibition of autophagy may be an effective way of improving antitumor strategies by enhancing apoptosis. In this study, we found that the combined treatment of NVP-BEZ235 and autophagy inhibition led to marked cell growth inhibition and increase of apoptosis. These results indicated that inhibition of autophagy likely impairs the proliferation mechanism of tumor cells, consequently leading to tumor cell growth inhibition. Furthermore, inhibition of autophagy increased the apoptotic marker caspase-3 activation, and thus led to the enhancement of tumor cell apoptosis. Indeed, previous studies have shown that inhibition of autophagy enhanced inhibitor-induced apoptosis (34-35). These findings are consistent with our hypothesis and indicate that autophagy inhibition could enhance the apoptosis of HCC cells and finally increase the growth inhibition of HCC cells. Thus, induction of autophagy by NVP-BEZ235 may be a survival mechanism that counteracts its anticancer effects. Based on these data, we suggest a strategy to enhance the anticancer efficacy of BEZ235 by blockade of autophagy.

In this study, we provided significant data indicating that inhibition of autophagy could enhance tumor cell growth inhibition and apoptosis of HCC cells induced by PI3K/mTOR inhibitor NVP-BEZ235. Thus, activation of autophagy may be involved in the resistance to apoptosis in HCC cells. Autophagy may help tumor cells mitigate metabolic stress and promote cell survival during apoptosis. In conclusion, we showed that inhibition of autophagy may be a novel way to increase the efficacy of antitumor strategies in the treatment of HCC.

\section{Acknowledgements}

This study was supported by the Basic Research Foundation of Jilin University, China (no. 201103048).

\section{References}

1. El-Serag HB and Rudolph KL: Hepatocellular carcinoma: epidemiology and molecular carcinogenesis. Gastroenterology 132: 2557-2576, 2007.

2. Siegel R, Naishadham D and Jemal A: Cancer statistics, 2012. CA Cancer J Clin 62: 10-29, 2012.

3. Verslype C, Van Cutsem E, Dicato M, et al: The management of hepatocellular carcinoma. Current expert opinion and recommendations derived from the 10th World Congress on Gastrointestinal Cancer, Barcelona, 2008. Ann Oncol 20 (Suppl 7): vii1-vii6, 2009.

4. Sia D and Villanueva A: Signaling pathways in hepatocellular carcinoma. Oncology 81 (Suppl 1): S18-S23, 2011.

5. Vivanco I and Sawyers CL: The phosphatidylinositol 3-Kinase AKT pathway in human cancer. Nat Rev Cancer 2: 489-501, 2002.

6. Luo J, Manning BD and Cantley LC: Targeting the PI3K-Akt pathway in human cancer: rationale and promise. Cancer Cell 4: 257-262, 2003.

7. Sabatini DM: mTOR and cancer: insights into a complex relationship. Nat Rev Cancer 6: 729-734, 2006.

8. Kong D and Yamori T: Advances in development of phosphatidylinositol 3-kinase inhibitors. Curr Med Chem 16: 2839-2854, 2009.

9. Pang RW and Poon RT: From molecular biology to targeted therapies for hepatocellular carcinoma: the future is now. Oncology 72 (Suppl 1): S30-S44, 2007.

10. Mizushima N, Levine B, Cuervo AM and Klionsky DJ: Autophagy fights disease through cellular self-digestion. Nature 451: 1069-1075, 2008.

11. Levine B and Kroemer G: Autophagy in the pathogenesis of disease. Cell 132: 27-42, 2008.

12. Rubinsztein DC, Gestwicki JE, Murphy LO and Klionsky DJ: Potential therapeutic applications of autophagy. Nat Rev Drug Discov 6: 304-312, 2007.

13. Masuda M, Shimomura M, Kobayashi K, et al: Growth inhibition by NVP-BEZ235, a dual PI3K/mTOR inhibitor, in hepatocellular carcinoma cell lines. Oncol Rep 26: 1273-1279, 2011.

14. Thomas HE, Mercer CA, Carnevalli LS, et al: mTOR inhibitors synergize on regression, reversal of gene expression, and autophagy in hepatocellular carcinoma. Sci Transl Med 4: 139-184, 2012.

15. Herrera VA, Zeindl-Eberhart E, Jung A, et al: The dual PI3K/ mTOR inhibitor BEZ235 is effective in lung cancer cell lines. Anticancer Res 31: 849-854, 2011. 
16. Shire AM and Roberts LR: Prevention of hepatocellular carcinoma: progress and challenges. Minerva Gastroenterol Dietol 58: 49-64, 2012.

17. Sandhu DS, Tharayil VS, Lai JP and Roberts LR: Treatment options for hepatocellular carcinoma. Expert Rev Gastroenterol Hepatol 2: 81-92, 2008.

18. Roberts LR and Gores GJ: Emerging drugs for hepatocellular carcinoma. Expert Opin Emerg Drugs 11: 469-487, 2006.

19. Tokunaga E, Oki E, Egashira A, et al: Deregulation of the Akt pathway in human cancer. Curr Cancer Drug Targets 8: 27-36, 2008.

20. Zou CY, Smith KD, Zhu QS, et al: Dual targeting of AKT and mammalian target of rapamycin: a potential therapeutic approach for malignant peripheral nerve sheath tumor. Mol Cancer Ther 8: $1157-1168,2009$.

21. Xu CX, Zhao L, Yue P, et al: Augmentation of NVP-BEZ235's anticancer activity against human lung cancer cells by blockage of autophagy. Cancer Biol Ther 12: 549-555, 2011.

22. Mirzoeva OK, Hann B, Hom YK, et al: Autophagy suppression promotes apoptotic cell death in response to inhibition of the PI3K-mTOR pathway in pancreatic adenocarcinoma. J Mol Med 89: 877-889, 2011

23. Jung CH, Jun CB, Ro SH, et al: ULK-Atg13-FIP200 complexes mediate mTOR signaling to the autophagy machinery. Mol Biol Cell 20: 1992-2003, 2009.

24. Fujiwara K, Iwado E, Mills GB, et al: Akt inhibitor shows anticancer and radiosensitizing effects in malignant glioma cells by inducing autophagy. Int J Oncol 31: 753-760, 2007.

25. Yang S, Xiao X, Meng X and Leslie KK: A mechanism for synergy with combined mTOR and PI3 kinase inhibitors. PLoS One 6: e26343, 2011.
26. Danial NN and Korsmeyer SJ: Cell death: critical control points. Cell 116: 205-219, 2004

27. Levine B and Klionsky DJ: Development by self-digestion: molecular mechanisms and biological functions of autophagy. Dev Cell 6: 463-477, 2004.

28. Mizushima $\mathrm{N}$ and Klionsky DJ: Protein turnover via autophagy: implications for metabolism. Annu Rev Nutr 27: 19-40, 2007.

29. Eisenberg-Lerner A, Bialik S, Simon HU and Kimchi A: Life and death partners: apoptosis, autophagy and the cross-talk between them. Cell Death Differ 16: 966-975, 2009.

30. Yang Y, Xing D, Zhou F and Chen Q: Mitochondrial autophagy protects against heat shock-induced apoptosis through reducing cytosolic cytochrome c release and downstream caspase-3 activation. Biochem Biophys Res Commun 395: 190-195, 2010.

31. Zhuang W, Qin Z and Liang Z: The role of autophagy in sensitizing malignant glioma cells to radiation therapy. Acta Biochim Biophys Sin 41: 341-351, 2009.

32. White E and DiPaola RS: The double-edged sword of autophagy modulation in cancer. Clin Cancer Res 15: 5308-5316, 2009.

33. Dikic I, Johansen T and Kirkin V: Selective autophagy in cancer development and therapy. Cancer Res 70: 3431-3434, 2010.

34. Nguyen TM, Subramanian IV, Xiao X, et al: Endostatin induces autophagy in endothelial cells by modulating Beclin 1 and betacatenin levels. J Cell Mol Med 13: 3687-3698, 2009.

35. Nguyen TM, Subramanian IV, Kelekar A and Ramakrishnan S: Kringle 5 of human plasminogen, an angiogenesis inhibitor, induces both autophagy and apoptotic death in endothelial cells. Blood 109: 4793-4802, 2007. 